\title{
Barriers in utilisation of low vision assistive products
}

\author{
Priya Sivakumar $^{1} \cdot$ Rajesh Vedachalam $^{2} \cdot$ Veena Kannusamy $^{3} \cdot$ Annamalai Odayappan $^{4} \cdot$ Rengaraj Venkatesh $^{5}$. \\ Pankaja Dhoble $^{6}$ - Fredrick Moutappa ${ }^{7}$ - Shivananda Narayana ${ }^{2}$
}

Received: 4 October 2018 / Revised: 16 July 2019 / Accepted: 26 July 2019 / Published online: 6 August 2019

(c) The Author(s), under exclusive licence to The Royal College of Ophthalmologists 2019

\begin{abstract}
Aim To understand the barriers in utilisation of low vision assistive products (LVAPs) from the perspective of patients with low vision.

Methods Patients referred to low vision clinic in a tertiary eye care hospital in India who were prescribed LVAPs but were un-willing to accept the products were interviewed using questionnaires. Data pertaining to the age, diagnosis, gender, occupation, preferred LVAPs, patient's perception of vision loss and the primary reason for non-acceptance of LVAPs were analysed.

Results A total of 235 among the 413 patients who noticed improvement in visual performance with LVAPs were unwilling to utilise these products. The questionnaire revealed that $53 \%$ of the patients who felt they were not candidates for LVAPs were experiencing severe visual impairment $(p<0.02)$. Non-acceptance was highest $(68.6 \%)$ in patients $<15$ years of age. The most common causes of non-acceptance were social stigma in patients $<40$ years $(41.3 \% ; p<0.0001)$, fear of loss of employment in patients $41-60$ years $(26.6 \% ; p<0.01)$ and low necessity in patients $>60$ years $(25 \% ; p<0.001)$. Denial of the magnitude of their illness was more common in patients above 60 years $(16.5 \%)$. Non-acceptance rate was lowest for macular disorders (39.6\%) and highest for retinitis pigmentosa (81\%). Among devices, hand and stand magnifiers had the lowest non-acceptance rate (41\%). Telescopes and electronic devices had the highest rate of non-acceptance (92\% and 89\%, respectively).

Conclusion Reasons for poor utilisation of LVAPs are multifactorial extending beyond affordability or accessibility. Knowledge of these barriers can help in creating content for awareness campaigns among patients, healthcare professionals and general society. Further research is necessary on the psychological and psycho social contributors to this process.
\end{abstract}

\section{Introduction}

Priya Sivakumar

Priya86sundaralakshmi@gmail.com

1 Department of Neuro ophthalmology and low vision services, Aravind eye care, Puducherry 605007, India

2 Department of Cornea, Aravind eye care, Puducherry 605007, India

3 DNB Ophthalmology, Department of Pediatric ophthalmology, Aravind eye care, Puducherry 605007, India

4 DNB Ophthalmology, Department of Glaucoma, Aravind eye care, Puducherry 605007, India

5 Department of Glaucoma, Aravind eye care, Puducherry 605007, India

6 Department of Retina, Aravind eye care, Puducherry 605007, India

7 Department of Pediatrics, Aravind eye care, Puducherry 605007, India
Vision loss expert group (VLEG) estimates 253 million people to be visually impaired worldwide. Approximately $89 \%$ of people who are visually impaired live in low and middle income countries (LMIC) [1]. Visual impairment is reported to be associated with poor quality of life and negative health outcomes [2,3]. There is also a direct relationship between vision impairment (VI) and having falls, sustaining fractures and increased mortality [4, 5]. People with visual impairment are at a higher risk for developing psychological problems, depression and anxiety [6]. In addition, there is loss of productivity and the time spent by caregivers' also is substantially increased as vision decreases, all of which leads to an increase in direct and indirect costs for the family [7]. Low vision assistive products (LVAPs) and vision rehabilitation would increase the quality of life in such patients apart from decreasing the dependence on family members and reducing the health 
care costs associated with secondary and tertiary disabilities caused by low vision. Studies report that the utilisation of low vision services is alarmingly low, not only in LMIC, but universally. Only $5-10 \%$ of people with low vision actually access these services [8].

This study analyses the patient-reported barriers in utilising LVAPs which might allow them to function more effectively in their everyday life.

\section{Materials and methods}

The study received ethical aproval by the Institutional Review Board of Aravind Eye Hospital. Informed consent was obtained from all the participants before enrolment.

\section{Study location}

Our study was conducted at the low vision clinic situated in the tertiary branch of an institute which includes seven subspecialities, three general ophthalmology units, a free unit and also provides primary and secondary eye care through vision centres and community centres in and around the district of Puducherry, South India. Patients can directly consult at the tertiary centre or can attend the secondary or primary centres and would be referred to tertiary centre when necessary. Criteria for low vision was defined as best corrected distance visual acuity $<6 / 18(0.50)$ in the better eye and/or near visual acuity (NVA) $<$ N10 and/or visual field $20^{\circ}$ or $<20^{\circ}$ from the point of fixation. Optometrists/ophthalmologists examining the patients in various sub-speciality clinics and general units assess and refer patients who fulfil the above criteria (whose vision needs are not met by routine refractive correction/medical or surgical management) to our low vision clinic headed by a low vision trained ophthalmologist and two optometrists.

\section{Study participants}

Patients evaluated at our low vision clinic who noticed improvement in visual performance with LVAPs $(\geq 3$ logMAR line improvement) but were unwilling to utilise these products due to various reasons were included as study participants. Patients with best corrected distance visual acuity in the better eye $<1 / 60$ or residual field less than five degrees around central fixation were excluded. Patients with low intellectual level or cognitive problems (defined as a score of $\leq 5$ on the mini mental scale examination) were also excluded. Age was no restriction.

\section{Classification of VI}

WHO-ICD-10 classification of VI is as follows:
Category 1-Moderate VI: Best corrected distance visual acuity in the better eye is worse than $6 / 18(0.50)$ and better than or equal to $6 / 60(1.00)$.

Number of study participants under this category- 72 .

Category 2-Severe VI: Best corrected distance visual acuity in the better eye is worse than 6/60 (1.00) and better than or equal to $3 / 60$ (1.30).

Number of study participants under this category- 118 .

Category 3 Blindness: Best corrected distance visual acuity in the better eye is worse than 3/60 (1.30) and better than or equal to $1 / 60(1.50)$ or the visual field of the better eye is $<10^{\circ}$ in radius around central fixation.

Category 4 Blindness: Best corrected distance visual acuity in the better eye is worse than 1/60 (1.50) and better than or equal to light perception.

Category 5 Blindness: No light perception.

As per the above classification, categories three to five come under blindness. As patients in category four and five benefit more from counselling, sensory substitution, mobility training, rehabilitation and environmental modification rather than LVAPs, which the current study focuses on, these patients are not included in our study. For the purpose of this study, instead of blindness, we have included a category-very severe VI.

Very severe VI: Best corrected distance visual acuity in the better eye is worse than $3 / 60$ (1.30) and better than or equal to $1 / 60(1.50)$ or patients who in addition to moderate or severe VI also have a disabling visual field defect (hemianopia/visual field of better eye $<20-5^{\circ}$ in radius around central fixation).

Number of study participants under this category- 45 .

\section{Clinical setting}

All participants were tested under binocular viewing conditions with recent habitual optical correction. Distance visual acuity as measured using an externally illuminated Lea numbers logMAR chart (Precision vision-745, North Harvard avenue, USA) at a distance of $4 \mathrm{~m}$. NVA was measured using Bailley-Lovie word reading chart and Snellen's NVA book for those familiar with the regional languages. Calculation of magnification required was done by the following formula.

Equivalent viewing power $=$ Best corrected visual acuity/target visual acuity $\times 100 /$ working distance

The LVAPs routinely offered in our clinic include:

i. High addition spectacles ( $>+4.00 \mathrm{DS})$ like convex sphere, aspheric sphere, prismospheres (prism glasses to utilise preferred retinal locus), aspheric lenticular, aspheric doublet and clip on loupes.

ii. Hand and stand magnifiers, pocket magnifiers, magnifiers with illumination 


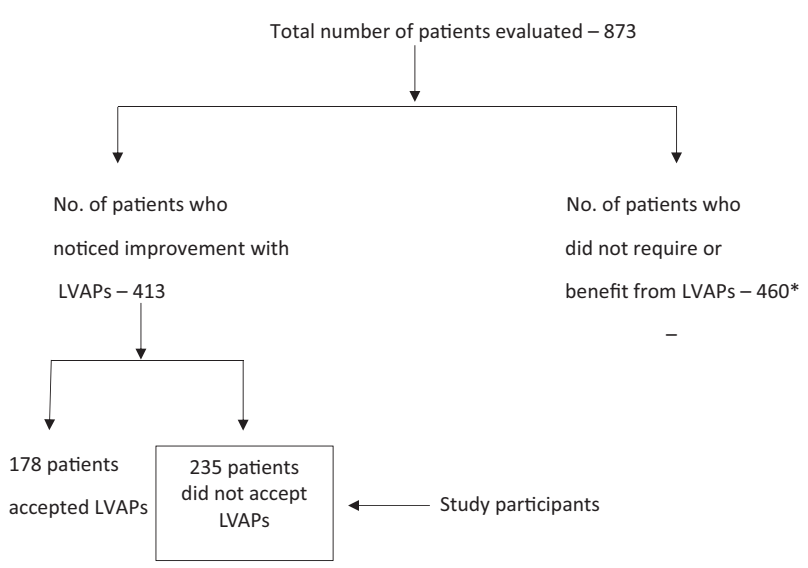

Fig. 1 Details of patients who presented to our low vision clinic during the study period. (Review patients are not included.) *Details of the remaining 460 patients who did not require or benefit from LVA trial: 139 patients improved with higher additions $(<+4.00 \mathrm{DS})$ in spectacles, not requiring LVA trial. 134 were patients with retinitis pigmentosa had well preserved central vision (6/6-6/9), referred to us for counselling. Around 63 were children $<5$ years of age with cortical visual impairment or retinopathy of prematurity who were referred for vision stimulation therapy and not for devices trial. Seventeen of our patients did not notice improvement with any sort of low vision device. Fifty-one patients had no form vision whom we referred to rehabilitation centres**. Thirty-six patients were mono ocular patients (functional/normal vision in the better eye) referred to us for disability certificate**. Total -873 patients. **Even though patients with no form vision should not be ideally referred to a low vision clinic, in our setup these patients are referred to low vision clinic for us to further guide them to their nearest blind school or rehabilitation centre. Our clinic also handles fitness or disability certification for mono ocular patients

iii. Telescopes-distance/near, hand held/spectacle mounted, monocular/binocular.

iv. Electronic devices-closed circuit TV and video magnifiers.

v. Non optical devices-signature card, notex, needle threader, typoscopes and mobility cane. These are available at subsidised rates (50\% concession) for purchase as soon as they are prescribed. Patients who were unwilling to utilise these LVAPs despite noticing improvement in visual performance ( $\geq 3 \log$ MAR line improvement) were interviewed with a questionnaire that explored the participant's perception of low vision and the primary reason for not accepting LVAPs. As no questionnaire exists to examine barriers in accepting LVAPs, these questions were developed by the authors. Pilot study was completed with 23 patients. Initially, patients were interviewed in the absence of their care-givers to give them privacy or a space to speak freely. After testing multiple scenarios, we found that our patients preferred the presence of family members during the interview. We requested the accompanying caregivers/family members to silently observe the patients' responses rather than prompting or giving opinions to prevent bias.

In paediatric patients ( $<15$ years), as parents are the main decision makers, they were given a choice to actively participate in the interview. If the child responded spontaneously to the questions, his or her response was recorded. If there was a contradiction between the parents and the child on the reason for rejection, they were encouraged to discuss among themselves and the final response was recorded. If the child did not respond, the parents' response was recorded.

We recorded multiple causes from the same patient initially, then we realised each patient did have a primary cause that was bothering him or her the most compared to other reasons. So, in the final questionnaire, the primary cause or the most important cause for refusal to use the device was recorded. (The cause which if negated, would allow the patient to use the device.)

Example-

"Question: Why would you not use this device?

Response: If my colleagues come to know that I cannot see, then I might lose my job. Also, I do not have enough money with me right now to buy this.

Question: If this device is given free of cost, would you use it?

Response: Well, that's fine. But I might still lose my job, so I prefer not to use it.

Question: If using the device does not endanger your job, would you use it?

Response: Yes, I would like to use it."

Even when more than one reasons are stated, there is one primary concern that is bothering the patient. He or she is willing to forego the other difficulties.

Patients had a free choice of reason for rejection. No list or options were provided. No leading questions were asked.

The questionnaire included the following questions:

1. Do you consider yourself as having permanent low vision?

2. Do you appreciate improvement in vision with any of these LVAP?

3. Which is your preferred LVAP?

4. Would you utilise the preferred LVAP in your daily activities?

If the answer to the above question is no,

5. What are your reasons for not choosing to use the LVAP?

If more than one response is recorded for the above question,

6. What is the primary/most important reason for not accepting the LVAP? 
Apart from the responses to questions, data pertaining to the age, gender, occupation, uncorrected and best corrected visual acuity and the diagnosis were also recorded.

\section{Data analysis}

The results from the questionnaire were analysed using SPSS statistical package (version 10; SPSS Science, Chicago, IL). Chi-square test and Fisher's exact test were used to identify differences in proportions. To analyse differences between the levels of VI and categorical variables of interest, a nonparametric Several Independent Samples test was used. A $p$ value of $<0.05$ was considered statistically significant.

\section{Results}

Details of the patients who attended our low vision clinic during the study period are depicted in Fig. 1.

Our study focuses on the 235 out of the 413 patients who noticed improvement in LVAPs trial but were unwilling to utilise LVAPs (non-acceptance rate: 56.9\%). Nonacceptance rate was highest in individuals $<40$ years of age (Table 1). Acceptance was relatively better in patients between 40 and 60 years. The male:female ratio of patients presenting to low vision clinic was 2.3:1. There was no significant difference in the acceptance/non-acceptance rate between the two groups.

Of the 235 participants, $29.3 \%$ had moderate VI, $48 \%$ had severe VI, and $18.3 \%$ had very severe VI based on the criteria mentioned above. 62 out of the 235 patients did not consider themselves as having low vision that requires LVA or did not understand the terminology based on their response to the questionnaire. Of these, 33 had severe or very severe VI (Fig. 2). This association was statistically significant $(p<0.027)$.

Non-acceptance rate was highest for telescopes $(92 \%)$ and electronic devices (89\%). It was lowest for hand \& stand magnifiers (41\%) and spectacle magnifiers (58\%) including prismospheres and aspheric glasses (Table 2).

The major causes of visual impairment were macular degeneration, retinitis pigmentosa, diabetic retinopathy, pathological myopia, optic atrophy, glaucoma, albinism, congenital nystagmus and congenital optic atrophy (Table 1). Non-acceptance rates were comparatively lower for retinal disorders affecting the central vision $(39.6 \%)$ like macular degeneration and diabetic retinopathy and were found to be higher for disorders predominantly affecting the visual fields like retinitis pigmentosa (81\%), high myopia/ hypermetropia (73\%), optic atrophy (64\%), and glaucoma $(50 \%)$.

The most common cause for non-acceptance among various age groups is shown in Table 3. Social stigma was
Table 1 Demographic characteristics and diagnoses of participants with low vision evaluated in this study

\begin{tabular}{|c|c|c|c|}
\hline Variables & Frequency & & \\
\hline 1. Age & $\begin{array}{l}\text { LVAP } \\
\text { recommended }\end{array}$ & $\begin{array}{l}\text { Accepted } \\
n(\%)\end{array}$ & $\begin{array}{l}\text { Non-accepted } \\
n(\%)\end{array}$ \\
\hline$<15$ years & 118 & $37(31.4)$ & $81(68.6)^{*}$ \\
\hline $16-40$ years & 109 & $40(36.7)$ & $69(63.3)^{*}$ \\
\hline $41-60$ years & 100 & $55(55)$ & $45(45)^{*}$ \\
\hline$>60$ years & 86 & $46(53.5)$ & $40(46.5)^{*}$ \\
\hline Total & 413 & $178(43.1)$ & $235(56.9)$ \\
\hline \multicolumn{4}{|l|}{ 2. Gender } \\
\hline Male & 287 & $128(44.5)$ & $159(55.4)$ \\
\hline Female & 126 & $50(39.6)$ & $76(60.4)$ \\
\hline \multicolumn{4}{|l|}{ 3. Patient profile } \\
\hline Students & 113 & 47 (41.6) & $66(58.4)$ \\
\hline Business & 52 & $36(69.3)$ & $16(30.7)$ \\
\hline Office/clerical work & 37 & $25(67.6)$ & $12(32.4)$ \\
\hline Daily wage/labourers & 36 & $14(39)$ & $22(61)$ \\
\hline Farmers & 48 & 19 (39.6) & $29(60.4)$ \\
\hline Home maker & 39 & $7(17.9)$ & $32(82.1)$ \\
\hline Retired personnel & 49 & $15(30.3)$ & $34(69.3)$ \\
\hline $\begin{array}{l}\text { Unemployed/not } \\
\text { attending school }\end{array}$ & 39 & $15(38.5)$ & $24(61.5)$ \\
\hline \multicolumn{4}{|l|}{ 4. Diagnosis } \\
\hline ARMD & 72 & $45(62.5)$ & $27(37.5)$ \\
\hline Retinitis pigmentosa & 70 & $14(20)$ & $56(80)$ \\
\hline Diabetic retinopathy & 59 & $39(66.1)$ & $20(33.9)$ \\
\hline High myopia/hypermetropia & 47 & $13(27.7)$ & $34(72.3)$ \\
\hline Optic atrophy/neuropathies & 41 & $16(39)$ & $25(61)$ \\
\hline Glaucoma & 40 & $20(50)$ & $20(50)$ \\
\hline Albinism & 31 & $9(29)$ & $22(71)$ \\
\hline Nystagmus $^{\mathrm{a}}$ & 27 & $12(44.4)$ & $15(55.6)$ \\
\hline Congenital optic nerve diseases & 26 & $10(38.5)$ & $16(61.5)$ \\
\hline
\end{tabular}

$A R M D$ age-related macular degeneration

*Significant association between different age groups and nonacceptance of LVAPs $(p<0.001)$

${ }^{\mathrm{a}}$ Other causes of nystagmus apart from albinism recorded in our study participants-cone rod dystrophy, post-trauma, post-intra cranial tumour excision, congenital nystagmus with low vision due to hypoxic ischaemic encephalopathy/autosomal dominant optic atrophy, strabismic amblyopia, retinopathy of prematurity sequelae and microphthalmos

the most common cause among patients up to 40 years of age. Fear of losing employment and usage difficulty in patients was the most common causes for patients between 41 and 60 years. Decreased necessity and acceptance of low vision as part of ageing were the leading causes in patients older than 60 years. Low affordability was stated as the primary cause of non-acceptance by $10.6 \%$ of the patients.

\section{Discussion}

LVAps play an integral role in vision rehabilitation. Harnessing the potential of assistive technology is a viable and achievable method of reducing the dependency of visually impaired individuals. If programs targeted at vision rehabilitation are to be effective, the reasons for poor utilisation of 


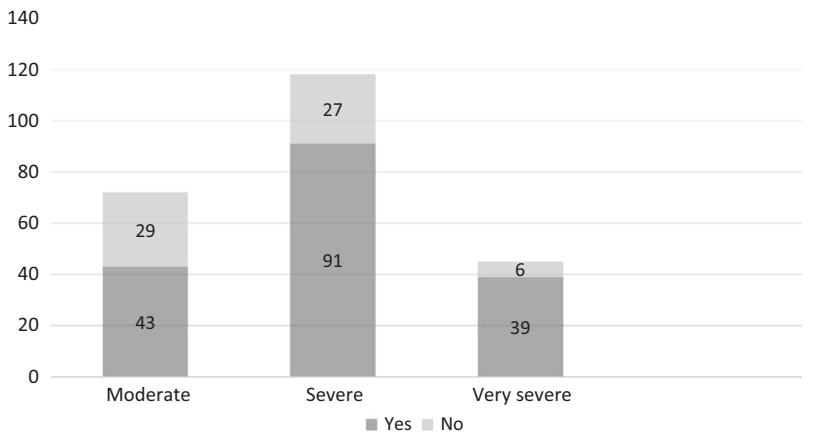

Fig. 2 Response to question,"Do you consider yourself as having permanent low vision?" depicted according to the degree of vision impairment

these products must be analysed. Previous studies have reported that there is a lack of awareness among eye care practitioners about low vision rehabilitation $[9,10]$. Very few studies have explored the patients' perspectives. We have attempted to address the patient-reported barriers in utilising LVAP in an Indian population.

Leading causes of low vision in our population were macular degeneration, retinitis pigmentosa and diabetic retinopathy. Uncorrected refractive errors were found to be the leading cause in a study by Wong et al. in East Asia [11]. Due to extensive school screening camps and outreach activities, uncorrected refractive errors have become less common in our area.

Non-acceptance rate was highest among patients with retinitis pigmentosa and high myopia/hypermetropia. This finding was anticipated, as 80-90\% of LVAPs users use their devices for near vision type reading tasks. Retinitis pigmentosa patients retain good central vision till advanced stages of the disease and myopic patients take their glasses off to read at a reduced working distance. In general, non-acceptance rates were lower for macular disorders (39\%) affecting the central vision and were higher for disorders affecting the visual fields like retinitis pigmentosa (81\%), optic atrophy (64\%), and glaucoma (50\%).

Barriers to accessing low vision rehabilitation services in a study by Pollard et al. (2003) revealed the general perception of rehabilitation in the population. Overcoming transportation hurdles, lack of awareness, and poor communication with eye-care professional were found to be the main concerns [12]. Transportation and physical access were also reported by O'Day et al. (2004) as leading barriers [13]. Interestingly, distance did not come up as a major barrier theme in the present study probably because our LVA clinic is functioning in the same premises of the hospital where the referring specialities are located. Similar results were reported in Quebec by Overbury O et al. [14]. Though in our region there is adequate connectivity by the public transportation system at a very nominal cost, distance as a barrier could not be

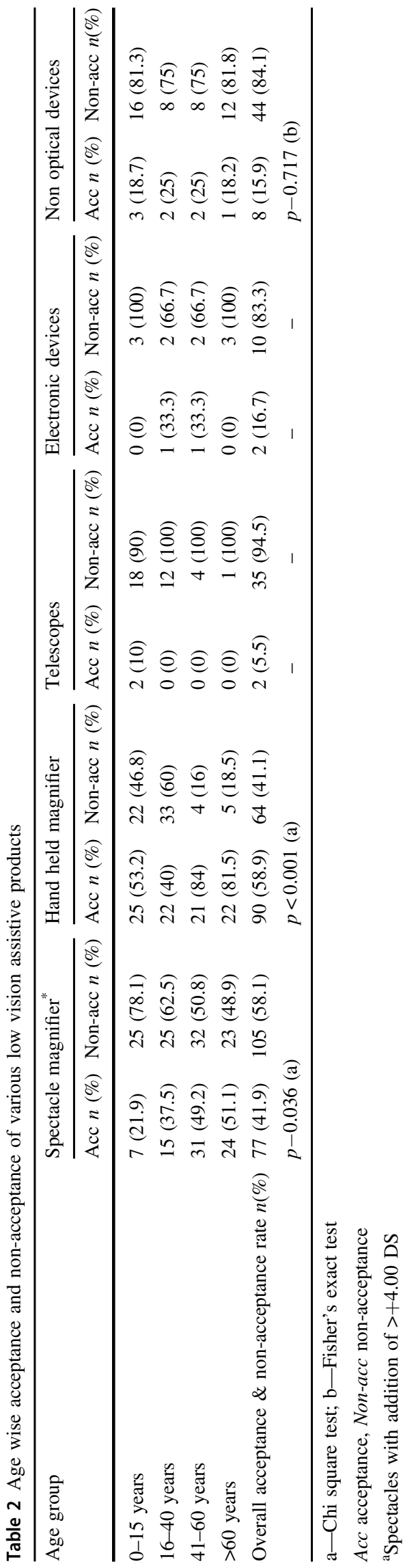


Table 3 Causes for nonacceptance of LVAPs across various age groups

\begin{tabular}{lclllc}
\hline Reasons/barriers & $<15$ years $\%$ & $\begin{array}{l}16-40 \text { years } \% \\
(n)\end{array}$ & $\begin{array}{l}41-60 \text { years } \% \\
(n)\end{array}$ & $\begin{array}{l}>60 \text { years } \% \\
(n)\end{array}$ & $\begin{array}{l}\text { Total } \% \\
(n)\end{array}$ \\
\hline Social stigma & $33.6(27)$ & $50.9(35)$ & $17.7(8)$ & $14.5(6)$ & $32.3(76)^{*}$ \\
Low awareness & $18.7(15)$ & $7(5)$ & $4.4(2)$ & $10.5(4)$ & $11(26)$ \\
Denial of magnitude & $9.8(8)$ & $7.1(5)$ & $8.9(4)$ & $16.5(6)$ & $9.7(23)$ \\
Fear of loss of job & 0 & $11 .(8)$ & $26.6(12)$ & $2.5(1)$ & $8(21)^{*}$ \\
Transportation (further & $4.9(4)$ & $5.9(4)$ & $6.8(3)$ & $11.5(5)$ & $6.8(16)$ \\
follow up) & $7(6)$ & 0 & $8.9(4)$ & $21.5(10)$ & $8.5(20)^{*}$ \\
Low necessity & $11.1(9)$ & $8.7(6)$ & $17.9(8)$ & $14(5)$ & $11.9(28)$ \\
Usage difficulty & $14.9(12)$ & $8.5(6)$ & $8.8(4)$ & $9(3)$ & $10.6(25)$ \\
Low affordability & 81 & 69 & 45 & 40 & 235 \\
Total & & & & & \\
\hline
\end{tabular}

* Significant association was noted between age group and cause of rejection: Less than 40 years-social stigma $(p<0.0001) ; 40-60$ years-fear of loss of employment $(p<0.01)$; $>60$ years-low necessity $(p<0.001)$ completely ruled out as we were unable to include the patients referred from primary and secondary centres who did not reach the tertiary base hospital.

Low affordability was stated as the primary cause of nonacceptance only by $10.5 \%$ of our patients. This is mainly because hospital provided financial aids covered $50 \%$ of the cost of any device that was recommended. It is concerning to note that despite the financial assistance, non-acceptance rate was still $56.9 \%$ in our population. It is worth mentioning that in spite of the subsidy provided, comparative remaining cost of certain LVAPs-electronic devices in particular like video magnifiers and CCTV were still unaffordable by majority of our patients.

Stigma is a powerful phenomenon with far-ranging effects on its targets. Stigma has been linked to poor mental health, physical illness, academic underachievement, low social status and poverty [15]. The leading cause of nonacceptance in our study in patients $<40$ years was social stigma.

Non-acceptance rate was highest $(69 \%)$ in the age group of $<15$ years followed by $63 \%$ in $15-40$ years. In the subset of patients $<15$ years, the majority of the decisions were made by the parents rather than the visionimpaired children and adolescents. Fear of rejection by society is so overwhelming among their parents, often resulting in non-acceptance. The second most common cause in this group was the lack of awareness of the benefits that could be gained with LVAPs.

Low vision is associated with an inability to continue gainful employment in the working age population [16]. Fear of loss of employment and difficulty in handling the devices were commonly reported in patients between 41 and 60 years, while overall non-acceptance rate was $45 \%$ in this group, which was relatively lower than other groups.
Among patients above 60 years, non-acceptance rate was $47 \%$. The majority of patients above 60 years stated that they found no necessity to use these devices as they are mostly indoors or dependent on caregivers. Some patients who did have improvement with magnifiers were still reluctant as they felt device use was time consuming and cumbersome.

Denial of magnitude of their illness was high in $>60$ years of age. Many patients above 60 years accepted low vision as part of aging process and felt it was unnecessary to adapt extra measures to overcome it.

In younger age groups of $<15$ years, many parents felt that the children would eventually gain vision as they grow. Awareness about childhood blindness or VI was overwhelmingly low. In general, patients with partial sight loss did not consider themselves "blind." A participant's fear of being perceived as blind has been reported as a major barrier in a study by Walter et al. (2008) [16]. Twenty-six percent of our patients who rejected LVAPs did not consider themselves as visually impaired and were reluctant to utilise services that seem geared towards the "blind." Misconceptions about visual impairment and blindness were frequent and pervasive. This clearly emphasises the need to educate the public on the vocabulary of VI and the purpose of vision rehabilitation.

The Montreal barriers study identified that even with full knowledge of the services and ideal referral conditions, only $56 \%$ actually entered the rehabilitation process. The study concluded that factors such as psychosocial or psychological characteristics may also play a significant role in the decision-making process of the individual [14]. We have attempted to explore the barriers in our population from patients' point of view. These findings highlight the myriad of perceptions patients with low vision and their family hold. Social stigma and misconceptions about low vision turned out to be the major hurdles in the acceptance of 
visual aids. As opposed to the existing assumption [17], poor resources and physical access are not the primary barriers. Understanding the root causes of low utilisation can help reduce the burden of VI. This information can be used in creating awareness among health care professionals, people with low vision and the general public. Lack of resources is not the leading barrier to vision rehabilitation with LVAPs. It is the patient's perceptions and misconceptions that increase the burden of blindness.

\section{Limitations}

Though our institute provides primary, secondary and tertiary care covering a wide geographical area and also is the sole low vision services providing clinic in the district of Puducherry with a population of 754,520 , only patients reaching the tertiary hospital were studied thus limiting the generalisability. Patients were interviewed but focus groups were not conducted which could have added qualitative value to the study. Apart from LVAPs, other aspects of vision rehabilitation are not covered in this study.

\section{Summary}

\section{What was known before}

- Low vision services are underutilized universally.

- Few quantitative studies have been done to understand the referral patterns and knowledge among health care professionals.

- Studies on patient reported barriers in utilising LVAPs are scarce and mainly focus on poor resource availability.

\section{What this study adds}

- In our study, analysis of patient reported barriers reveal that in spite of resource availability, acceptance of LVAPs is alarmingly low.

- Stigma, fear of loss of employment and low necessity are the leading barriers.

- This information can be used in developing contents for awareness campaigns among patients and doctors.

- By addressing the root causes of underutilisation of vision rehabilitation, the burden of blindness can be reduced.

\section{Data availability}

All data are reported in the manuscript.

Acknowledgements Authors sincerely thank Mrs. M. Iswarya and Mr. K. Balagiri, Department of Biostatistics, Aravind eye care,
Madurai for their help in statistical analysis, Miss. Olivia M. Hess for helping with language edits, Mr. Pari and Miss. Esha, our low vision services users for more than past 7 years for their inputs and Miss. Flora, low vision rehabilitationist for her support.

Funding This study was supported by Mahima foundation and Aravind eye care system, India.

\section{Compliance with ethical standards}

Conflict of interest The authors declare that they have no conflict of interest.

Ethics approval Institutional research and ethical committee approval was obtained from Aravind Eye care, Madurai, Tamil Nadu, India.

Informed consent Written informed consent has been obtained from all participants of this study.

\section{References}

1. Bourne RRA, Flaxman SR, Braithwaite T, Cicinelli MV, Das A, Jonas JB, et al. Magnitude, temporal trends, and projections of the global prevalence of blindness and distance and near vision impairment: a systematic review and meta-analysis. Lancet Glob Health. 2017;5:e888-97.

2. Chia E-M, Mitchell P, Ojaimi E, Rochtchina E, Wang JJ Assessment of vision-related quality of life in an older population subsample: The Blue Mountains Eye Study. Ophthalmic Epidemiol. 2006;13:371-7.

3. Langelaan M, de Boer MR, van Nispen RMA, Wouters B, Moll AC, van Rens GHMB. Impact of visual impairment on quality of life: a comparison with quality of life in the general population and with other chronic conditions. Ophthalmic Epidemiol. 2007;14:119-26.

4. Lord SR. Visual risk factors for falls in older people. Age Ageing. 2006;35:ii42-ii45.

5. Felson DT, Anderson JJ, Hannan MT, Milton RC, Wilson PW, Kiel DP. Impaired vision and hip fracture. The Framingham Study. J Am Geriatr Soc. 1989;37:495-500.

6. Kempen GIJM, Ballemans J, Ranchor AV, van Rens GHMB, Zijlstra GAR. The impact of low vision on activities of daily living, symptoms of depression, feelings of anxiety and social support in community-living older adults seeking vision rehabilitation services. Qual Life Res. 2012; 21:1405-11.

7. Köberlein J, Beifus K, Schaffert C, Finger RP. The economic burden of visual impairment and blindness: a systematic review. BMJ Open. 2013;3:e003471.

8. Chiang PP-C, O'Connor PM, Le Mesurier RT, Keeffe JE. A global survey of low vision service provision. Ophthalmic Epidemiol. 2011;18:109-21.

9. National Eye Institute. Low Vision Focus Groups Final Report: ophthalmologists, optometrists, and office staff. 2001. http://www. nei.nih.gov/nehep/pro_focus.htm. Accessed 3 Mar 2002.

10. Keeffe JE, Lovie-Kitchin JE, Taylor HR. Referral to low vision services by ophthalmologists. Aust N Z J Ophthalmol. 1996;24: 207-14.

11. Wong TY, Zheng Y, Jonas JB, Flaxman SR, Keeffe J, Leasher J, et al. Prevalence and causes of vision loss in East Asia: 1990-2010. Br J Ophthalmol. 2014;98:599-604. 
12. Pollard TL, Simpson JA, Lamoureux EL, Keeffe JE. Barriers to accessing low vision services. Ophthalmic Physiol Opt. 2003;23:321-7.

13. O'Day BL, Killeen M, Iezzoni LI. Improving health care experiences of persons who are blind or have low vision: suggestions from focus groups. Am J Med Qual. 2004;19:193-200.

14. Overbury $\mathrm{O}$, Wittich W. Barriers to low vision rehabilitation: the Montreal Barriers Study. Invest Ophthalmol Vis Sci. 2011;52:8933-8.
15. Major B, O'Brien LT. The social psychology of stigma. Annu Rev Psychol. 2005;56:393-421.

16. Walter C, Althouse R, Humble H, Leys M, Odom J. West Virginia survey of visual health: low vision and barriers to access. Vis Impair Res. 2004;6:53-71.

17. van Dijk K. Definition: visual impairment. In: Punani B, Rawal N, editors. Visual impairment and blindness. Chap. 1, vol. 1. Vastrapur, Ahmedabad: Blind People's Association; 2000. p. $1-10$. 